\title{
Liver Cancer Disparities in New York City: A Neighborhood View of Risk and Harm Reduction Factors
}

\author{
Geetanjali R. Kamath ${ }^{1,2,3}$, Emanuela Taioli1,2,3, Natalia N. Egorova ${ }^{3}$, Josep M. Llovet ${ }^{4,5,6}$, \\ Ponni V. Perumalswami ${ }^{7}$, Jeffrey J. Weiss ${ }^{8}$, Myron Schwartz ${ }^{9}$, Stanley Ewala ${ }^{10}$ \\ and Nina A. Bickell ${ }^{2,3,8 *}$
}

\begin{abstract}
${ }^{1}$ Institute for Translational Epidemiology, New York, NY, United States, ${ }^{2}$ Tisch Cancer Institute, New York, NY, United States, ${ }^{3}$ Department of Population Health Science and Policy, Icahn School of Medicine at Mount Sinai, New York, NY, United States, ${ }^{4}$ Mount Sinai Liver Cancer Program, Divisions of Liver Diseases, Icahn School of Medicine at Mount Sinai, Tisch Cancer Institute, New York, NY, United States, ${ }^{5}$ Liver Cancer Translational Research Laboratory, BCLC, Liver Unit, Centro de Investigación Biomédica en Red de Enfermedades Hepáticas y Digestivas (CIBEREHD), Institut d'IInvestigacions Biomèdiques August Pi iSunyer (IDIBAPS), University of Barcelona, Barcelona, Catalonia, Spain, ${ }^{6}$ Institució Catalana de Recerca i Estudis Avançats, Barcelona, Catalonia, Spain, ${ }^{7}$ Division of Liver Diseases, Department of Medicine, Icahn School of Medicine at Mount Sinai, New York, NY, United States, ${ }^{8}$ Division of General Internal Medicine, Department of Medicine, Icahn School of Medicine at Mount Sinai, New York, NY, United States, ${ }^{9}$ Department of Surgery, Icahn School of Medicine at Mount Sinai, New York, NY, United States, ${ }^{10} / c a h n$ School of Medicine at Mount Sinai, New York, NY, United States
\end{abstract}

OPEN ACCESS

Edited by:

Friederike Erdmann,

Danish Cancer Society, Denmark

Reviewed by:

Jerry Polesel,

Centro di Riferimento Oncologico di Aviano (IRCCS), Italy Mary H. Ward,

National Cancer Institute (NCl), United States

*Correspondence: Nina A. Bickell nina.bickel/@mssm.edu

Specialty section: This article was submitted to Cancer Epidemiology and Prevention, a section of the journal

Frontiers in Oncology

Received: 10 April 2018 Accepted: 29 May 2018 Published: 14 June 2018

Citation:

Kamath GR, Taioli E, Egorova NN,

Llovet JM, Perumalswami PV,

Weiss JJ, Schwartz M, Ewala S and Bickell NA (2018) Liver Cancer

Disparities in New York City: A Neighborhood View of Risk and Harm Reduction Factors.

Front. Oncol. 8:220. doi: 10.3389/fonc.2018.00220
Introduction: Liver cancer is the fastest increasing cancer in the United States and is one of the leading causes of cancer-related death in New York City (NYC), with wide disparities among neighborhoods. The purpose of this cross-sectional study was to describe liver cancer incidence by neighborhood and examine its association with risk factors. This information can inform preventive and treatment interventions.

Materials and methods: Publicly available data were collected on adult NYC residents ( $n=6,407,022)$. Age-adjusted data on liver and intrahepatic bile duct cancer came from the New York State Cancer Registry (1) (2007-2011 average annual incidence); and the NYC Vital Statistics Bureau (2015, mortality). Data on liver cancer risk factors (2012-2015) were sourced from the New York City Department of Health and Mental Hygiene: (1) Community Health Survey, (2) A1C registry, and (3) NYC Health Department Hepatitis surveillance data. They included prevalence of obesity, diabetes, diabetic control, alcohol-related hospitalizations or emergency department visits, hepatitis B and $\mathrm{C}$ rates, hepatitis $\mathrm{B}$ vaccine coverage, and injecting drug use.

Results: Liver cancer incidence in NYC was strongly associated with neighborhood poverty after adjusting for race/ethnicity $(\beta=0.0217, p=0.013)$; and with infection risk scores $(\beta=0.0389,95 \% \mathrm{Cl}=0.0088-0.069, p=0.011)$, particularly in the poorest neighborhoods ( $\beta=0.1207,95 \% \mathrm{Cl}=0.0147-0.2267, p=0.026$ ). Some neighborhoods with high hepatitis rates do not have a proportionate number of hepatitis prevention services.

Conclusion: High liver cancer incidence is strongly associated with infection risk factors in NYC. There are gaps in hepatitis prevention services like syringe exchange and vaccination that should be addressed. The role of alcohol and metabolic risk factors on liver cancer in NYC warrants further study.

Keywords: hepatocellular carcinoma, chronic hepatitis, health-care disparities, low-income populations, vaccinations, cancer screening 


\section{INTRODUCTION}

Cancer of the liver and intrahepatic bile ducts (liver cancer) is a public health problem in the United States (US). Since 1980, its nationwide incidence rate has tripled, and its mortality rate has doubled, outpacing the increase in any other cancer (2). Only $31 \%$ of those with localized liver cancer survive 5 years past diagnosis. The 5-year survival rate for regional and distant liver cancer is even poorer at 11 and 3\%, respectively (2). Several studies have succeeded in reducing liver cancer incidence, most effectively through hepatitis B vaccination, and to an extent through antiviral therapy for hepatitis B and C (3). Although liver cancer prognosis can be improved by early detection and treatment during its long subclinical course, this is a challenge since liver cancer is usually asymptomatic in its early stages $(3,4)$. Currently, no guidelines currently exist for routine liver cancer screening in people of average risk; however, people at higher risk due to cirrhosis and/ or chronic hepatitis B infection may benefit from screening with ultrasound exams, with or without alpha-fetoprotein blood tests, twice a year (5).

In New York City (NYC), liver cancer is the fifth leading cause of cancer-related death among men and seventh among women (1). The 2010-2014 age-adjusted liver cancer incidence rate in NYC was 12 per 100,000 residents, higher than the US (7.8) and New York State (NYS) $(8.6)(1,6)$. The age-adjusted mortality rate per 100,000 was also higher at 7.7 compared with the US (6.3) and NYS $(6.1)(1,6)$. Certain neighborhoods have incidence rates of 16-22.2 per 100,000, comparable to Asia, West Africa, and Central/South America (7).

New York City is a microcosm of the global population due to its unique demographics, high percentage of foreign-born inhabitants, and diversity of country of origin. Although a recent review examined racial/ethnic liver cancer disparities in the US (8), it has not been studied on a local level. A study of cancer incidence in NYC and three of its neighborhoods (East Harlem, Central Harlem, and Upper East Side) found that neighborhood was associated with incidence of all cancers, including liver cancer (9). To understand the basis for NYC disparities in liver cancer incidence and mortality, it is crucial to identify high-risk subpopulations, the risk factors most strongly associated with liver cancer, and how they are distributed in the city. The information can help inform preventive and treatment interventions for communities that require them the most.

\section{MATERIALS AND METHODS}

\section{Data Collection}

Data were collected from the pool of adult ( $\geq 18$ years) NYC residents ( $n=6,407,022$ per the 2010 US Census) at the neighborhood level, and defined neighborhood borders using NYC United Hospital Fund (UHF) codes. Originally, the UHF divided NYC into 42 distinct neighborhoods by combining adjoining zip codes areas with similar characteristics, meant to approximate NYC Community Planning Districts. To increase statistical power, these were later collapsed into 34 neighborhoods (10).

\section{Primary Outcomes}

Cancer data for NYC included age-adjusted incidence and mortality rates per 100,000 residents from the NYS Cancer Registry (1). The average incidence rate was calculated from the number of residents diagnosed with liver and/or intrahepatic bile duct cancer over 2007-2011, divided by the corresponding age-specific intercensal population estimates (from the NYS Department of Health). Age adjustment was based on the US Census 2000 standard population. Mortality rate estimates for 2015 were obtained from the NYC Department of Health and Mental Hygiene (NYCDOHMH) using the online interactive tool, Epiquery (11). Crude mortality rates are presented for neighborhoods with small numbers and/or unreliable age-adjusted estimates. For all outcomes, the most recent available estimates at the neighborhood level are presented.

\section{Sociodemographics}

Data on gender and race/ethnicity were collected from the 2010 US Census, and neighborhood-specific distributions were extracted using Epiquery. Data on poverty were obtained from the American Community Survey conducted by the US Census Bureau (12). Poverty was defined as the $\%$ of people reporting annual incomes below the federal poverty threshold during 2010-2014 (\$11,139-\$12,071 for one person). Data on insurance coverage were obtained from the Community Health Survey (CHS), an annual telephone survey conducted among NYC residents $\geq 18$ years by the NYCDOHMH (13). We report the $\%$ of people who had no type of health insurance coverage.

\section{Risk Factors for Liver Cancer}

Viral hepatitis data are derived from surveillance reports filed by the Bureau of Communicable Disease (14). They include confirmed or probable cases of chronic hepatitis B and C reported to the Health Department by health-care providers and laboratories meeting the definitions by the Centers for Disease Control/ Council of State and Territorial Epidemiologists' (positive hepatitis B surface antigen, hepatitis B e-antigen, and hepatitis B nucleic acid test; enzyme-linked immunosorbent assay antibody test with a high signal-to-cutoff value; recombinant immunoblot assay; and RNA test for hepatitis C).

Prevalence of self-reported current smoking (proportion of people who reported smoking cigarettes daily or on some days as of the interview day), injecting drug use (\% of people who reported having used a needle to inject non-prescription drugs at least once), obesity (body mass index $\geq 30 \mathrm{~kg} / \mathrm{m}^{2}$ ), diabetes ( $\%$ of people who reported ever being told by a health-care professional that they have diabetes), and physical activity ( $\%$ of adults who reported in the past 30 days: (1) exercising (running, calisthenics, golf, gardening, or walking, other than at their regular job) and (2) walking/bicycling $>10$ blocks for transportation) were obtained via the CHS (13). Diabetes control was measured by data from the NYC A1C Registry. We report the $\%$ of diabetic adults (history of $\geq 2$ glycosylated hemoglobin, or A1C, test values $\geq 6.5 \%$ ) who received medical care in 2012 , with their last A1C measurement $\geq 9 \%$ (15).

As a proxy for alcohol use, clinical data on the number of patients who werehospitalized or visited an emergency department 
(ED) during 2014 were abstracted from the mandatory NYS hospital discharge abstract database (16), using the ICD-9 codes 291.0-291.5, 291.8, 291.9, 303.00-303.93, 305.00-305.03, 357.5, $425.5,535.3,571.1-4,571.5,571.9,572.3,577.1$ (diagnoses of alcohol-related morbidity) (17), and of alcohol poisoning (790.3, 980, E860) (18). Only one hospitalization/ED visit per patient was counted. Approval to collect data under exempt status was obtained from Mount Sinai's Institutional Review Board.

\section{Preventive Services}

Data on the availability of preventive services providing hepatitis $\mathrm{B}$ or $\mathrm{C}$ testing and treatment, hepatitis $\mathrm{B}$ vaccination, and syringe exchange facilities were collected from the NYC Health Map website (19), which lists names and addresses of clinics by service type. The number of services in each UHF neighborhood was obtained by matching address zip codes. The $\%$ of NYC residents who reported ever having received at least 1 dose of the hepatitis $B$ vaccine and ever getting tested for hepatitis $C$ was obtained from the CHS.

\section{Statistical Analyses}

Descriptive data are presented for the entire city, and each neighborhood in the form of tables and density maps prepared using ArcGIS Desktop (version 10.3.1; ESRI, Redlands, CA). Predictors were weighted risk scores calculated for three domains of modifiable liver cancer risk factors: (1) metabolic (obesity, diabetes, and proportion of $\mathrm{A} 1 \mathrm{C} \geq 9 \%$ ); (2) alcohol-related morbidity (hospitalizations, ED visits); and (3) infections (rates of newly reported hepatitis $\mathrm{B}$ and $\mathrm{C}$ cases, hepatitis $\mathrm{B}$ vaccination coverage, and self-reported injecting drug use). Each continuous item was given an ordinal score based on tertiles, quartiles, or a specific cutoff. For each item, neighborhoods received a prevalence score from 1 to 3 based on increasing tertiles (quartiles 1-4 for hepatitis B). Hepatitis B vaccine coverage was reverse scored to reflect a protective effect. Due to the distribution, a cutoff of $<1 \%$ and $\geq 1 \%$ was used to score injecting drug use prevalence category as 1 or 2. Each item was also assigned a correlation score from 1 to 3 based on the strength of its correlation with liver cancer incidence (Pearson's $r \leq 0.3,0.3<r<0.5, r \geq 5$ ). Prevalence scores were multiplied by the correlation scores to obtain item scores, which were summed up to produce a risk score for each domain.

Spatial autocorrelation of liver cancer incidence was assessed using Moran's global index (Moran's I statistic) (20). A sensitivity analysis was conducted to evaluate the effect of spatial dependence by comparing linear regression models with and without a spatial lag term. The spatial lag model was run by adding a spatial weights matrix as an independent variable with weights based on inverse distances between neighborhood centroid coordinates. All spatial analyses were conducted using the spatial software GeoDa version 1.12.1.129.

The relationship between liver cancer incidence and each predictor was assessed in unadjusted and adjusted generalized linear regression models, with neighborhood as the unit of analysis $(n=34)$ (SAS Proprietary Software 9.4, TS1M1). All models met the assumptions for the specified Poisson distribution (21). Stratified analyses by prevalence of neighborhood poverty were conducted. Point estimates, 95\% Wald confidence intervals, and $p$-values for the regression coefficient $\beta$ were evaluated at a statistical significance level of $\alpha=0.05$ (two-sided hypothesis test).

\section{RESULTS}

New York City's racial and ethnic composition includes 33\% non-Hispanic White, 29\% Hispanic, 23\% African-American, $13 \%$ Asian or Pacific Islander, and 3\% other races. Half of NYC residents $(53 \%)$ are female, $21 \%$ live in poverty, and $13 \%$ are uninsured. There was considerable variation in the distribution of demographic characteristics according to neighborhood (Table 1).

\section{Liver Cancer Statistics}

During 2007-2011, there was an average of 921.4 cases of liver and intrahepatic bile duct cancer in NYC annually. The age-adjusted incidence rates were highest in the Bronx [South Bronx (22.3), Fordham/Bronx Park (15), and Pelham/Throgs Neck (13.7)]; Manhattan [Union Square and Lower Manhattan (15.9), Central Harlem (15.8), East Harlem (15.7), and Washington Heights/ Inwood (13.3)], and Brooklyn [Sunset Park (16.7)] (Figure 1). Mortality rates follow similar geographic distribution, with the highest mortality rates in Sunset Park (12.6), Fordham/Bronx Park (12.1), South Bronx (11.6), Union Square and Lower Manhattan (11.5), Pelham/Throgs Neck (11), and Central Harlem (9.2). Two neighborhoods showed high mortality rates despite relatively lower incidence: Williamsburg/Bushwick in Brooklyn (10.6) and Ridgewood/Forest Hills in Queens (9.1).

\section{Liver Cancer Risk Factors}

The distribution of individual liver cancer risk factors is presented in Table 2. Obesity was less prevalent in NYC (24\%) compared with the US average $(\approx 38 \%)$ (4) but varied widely from $8 \%$ in the Upper West Side to 37\% in East New York. East Harlem had the highest prevalence of self-reported diabetes (23\%) and poor glycemic control (21\%). A high proportion of poorly controlled diabetes was also observed in East New York, Bedford-Stuyvesant/ Crown Heights, Williamsburg/Bushwick, the South Bronx, and Fordham/Bronx Park. There was relatively less variation in selfreported physical activity. East Harlem had the highest prevalence of self-reported injecting drug use at $4.7 \%$, followed by Upper West Side (2.1\%), and the South Bronx (1.8\%). Cigarette smoking was most prevalent in Greenpoint (21\%), Long Island City/Astoria, and Ridgewood/Forest Hills (19\%). Finally, the mean and range of composite scores for the three modifiable risk factor domains (metabolic, alcohol, and infection) are presented in Table 3. Alcohol risk scores were moderately correlated with metabolic and infection risk scores; however, results of statistical tolerance tests did not indicate a significant threat of multicollinearity on the model estimates (22).

\section{Association Between Distribution of Liver Cancer Incidence and Risk Factor Scores}

Neighborhood-level data on poverty and Hispanic ethnicity were associated with high liver cancer incidence $(\beta=0.0277$, $p<0.0001$, and $\beta=0.0113, p<0.0001$ ), even after adjustment 
TABLE 1 | Distribution of sociodemographic characteristics according to neighborhood.

\begin{tabular}{|c|c|c|c|c|c|c|c|c|}
\hline \multirow[t]{2}{*}{ Neighborhood } & \multirow[t]{2}{*}{$\%$ Male $^{a}$} & \multicolumn{5}{|c|}{ Race/ethnicity (\% of population) ${ }^{a}$} & \multirow[t]{2}{*}{$\%$ Living in poverty ${ }^{b}$} & \multirow[t]{2}{*}{$\%$ Uninsured $^{c}$} \\
\hline & & White & Black & Hispanic & Asian/Pacific Islander & Other & & \\
\hline Kingsbridge/Riverdale & 45.0 & 42.5 & 11.1 & 39.8 & 4.7 & 1.9 & 16.1 & 2.7 \\
\hline The Northeast Bronx & 44.7 & 11.1 & 58.8 & 24.4 & 2.8 & 2.9 & 15.4 & 10.3 \\
\hline Fordham/Bronx Park & 47.4 & 8.7 & 24.8 & 59.6 & 5.0 & 2.0 & 32.9 & 18 \\
\hline Pelham/Throgs Neck & 47.0 & 20.4 & 20.7 & 49.7 & 6.6 & 2.7 & 23.2 & 11.8 \\
\hline South Bronx ${ }^{d}$ & 46.9 & 1.5 & 29.5 & 66.5 & 1.0 & 1.4 & 41.2 & 11.9 \\
\hline Greenpoint & 49.5 & 68.1 & 2.9 & 23.0 & 4.1 & 1.9 & 26.5 & 8.6 \\
\hline Downtown Brooklyn/Heights/Slope & 47.1 & 56.6 & 15.5 & 18.1 & 6.5 & 3.3 & 16.4 & 10.3 \\
\hline Bedford-Stuyvesant/Crown Heights & 44.8 & 11.2 & 71.4 & 13.1 & 1.9 & 2.5 & 27.2 & 11.4 \\
\hline East New York/New Lots & 46.1 & 1.9 & 51.2 & 38.8 & 4.7 & 3.4 & 33.4 & 3.8 \\
\hline Sunset Park & 51.4 & 15.8 & 2.3 & 44.6 & 35.7 & 1.6 & 31.1 & 27.4 \\
\hline Borough Park & 49.4 & 61.0 & 4.3 & 12.8 & 20.0 & 1.8 & 26.9 & 16.0 \\
\hline Flatbush & 45.0 & 11.9 & 72.4 & 10.9 & 2.3 & 2.4 & 18.6 & 14.3 \\
\hline Canarsie and Flatlands & 44.4 & 24.1 & 61.4 & 8.9 & 3.5 & 2.1 & 13.3 & 8.5 \\
\hline Bay Ridge/Bensonhurst & 48.5 & 60.3 & 1.1 & 13.4 & 23.4 & 1.8 & 16.0 & 13.2 \\
\hline Coney Island & 47.2 & 64.7 & 6.6 & 11.7 & 15.4 & 1.6 & 20.4 & 11.3 \\
\hline Williamsburg/Bushwick & 48.5 & 14.6 & 30.4 & 48.7 & 4.5 & 1.8 & 31.5 & 10.4 \\
\hline Washington Heights/Inwood & 48.0 & 15.9 & 12.0 & 68.0 & 2.5 & 1.7 & 25.4 & 18.7 \\
\hline Central Harlem & 45.6 & 13.9 & 54.6 & 24.2 & 4.3 & 2.9 & 29.8 & 5.3 \\
\hline East Harlem & 47.1 & 11.7 & 29.0 & 51.7 & 5.6 & 2.0 & 32.9 & 14.7 \\
\hline Upper West Side & 45.8 & 67.2 & 7.5 & 14.9 & 7.9 & 2.5 & 11.6 & 7.8 \\
\hline Upper East Side-Gramercy ${ }^{d}$ & 45.0 & 75.5 & 3.4 & 7.4 & 11.5 & 2.2 & 8.4 & 7.8 \\
\hline Chelsea Village $^{d}$ & 50.4 & 66.0 & 4.0 & 10.6 & 16.6 & 2.8 & 11.0 & 12.2 \\
\hline Union Sq-Lower Manhattan ${ }^{d}$ & 48.1 & 45.7 & 6.8 & 10.0 & 50.4 & 8.0 & 16.1 & 5.2 \\
\hline Long Island City/Astoria & 49.4 & 46.9 & 6.1 & 27.1 & 16.6 & 3.3 & 16.7 & 9.6 \\
\hline West Queens & 51.9 & 16.1 & 5.9 & 51.4 & 24.5 & 2.1 & 19.2 & 29.0 \\
\hline Flushing/Clearview & 47.7 & 31.3 & 2.1 & 16.2 & 48.4 & 1.9 & 15.2 & 12.8 \\
\hline Bayside-Fresh Meadows ${ }^{d}$ & 47.5 & 44.0 & 5.2 & 12.2 & 36.2 & 2.3 & 11.6 & 7.5 \\
\hline Ridgewood/Forest Hills & 47.7 & 54.5 & 2.0 & 26.1 & 15.5 & 2.0 & 13.1 & 17.6 \\
\hline Southwest Queens & 49.0 & 22.6 & 12.4 & 32.7 & 20.2 & 12.1 & 14.3 & 9.5 \\
\hline Jamaica & 46.7 & 7.1 & 53.9 & 18.0 & 14.5 & 6.5 & 16.0 & 19.6 \\
\hline Southeast Queens & 46.4 & 13.6 & 54.9 & 11.8 & 14.7 & 5.1 & 7.6 & 9.5 \\
\hline The Rockaways & 47.0 & 35.2 & 38.8 & 21.0 & 2.3 & 2.7 & 20.2 & 8.4 \\
\hline Northern Staten Island ${ }^{d}$ & 48.5 & 40.2 & 21.0 & 28.9 & 7.5 & 2.4 & 20.4 & 10.1 \\
\hline Southern Staten Island ${ }^{d}$ & 48.4 & 76.0 & 2.5 & 11.1 & 8.8 & 1.5 & 7.7 & 4.1 \\
\hline NYC & 47.5 & 33.3 & 22.8 & 28.6 & 12.6 & 2.7 & 20.6 & 12.6 \\
\hline
\end{tabular}

anited States Census, 2010.

bAmerican Community Survey, percentage with annual income below 100\% of federal poverty threshold, 2010-2014.

'New York City (NYC) Department of Health and Mental Hygiene. Epiquery: NYC Interactive Health Data System-[Community Health Survey 2015] [08/28/2017].

http://nyc.gov/health/epiquery.

${ }^{\circ}$ Gender, race, and poverty data for combined neighborhoods are averages of constituent UHF 42 neighborhoods.

All percentages are age-adjusted using the 2010 US Census standard population.

for White race and Hispanic ethnicity $(\beta=0.0217, p=0.013)$. A higher proportion of foreign-born residents was correlated with higher rates of hepatitis $\mathrm{B}(r=0.48, p=0.0037)$.

Among the three modifiable risk factor domains, infection was the strongest predictor of liver cancer incidence, with an expected increase of $5.3 \%$ in incidence when the infection risk score increased by $1(p<0.0001)$, followed by alcohol-related morbidity ( $4.8 \%$ increase, $p=0.001$ ) (Table 3 ). Metabolic risk score was also weakly but positively associated with liver cancer incidence ( $3 \%$ increase, $p=0.052$ ). We conducted formal testing by including interaction terms between poverty tertiles and each of the three risk scores (metabolic, alcohol, and infection), and observed lack of statistical interaction. When stratified by tertiles of poverty prevalence, infection score was most strongly associated with liver cancer incidence at the high poverty level ( $10 \%$ increase, $p=0.027$ ). Similarly, infection risk score was most strongly associated with liver cancer incidence (4\% increase, $p=0.011)$, especially at the high poverty level (12.8\% increase, $p=0.026)$, in models that adjusted for metabolic and alcohol risk score.

\section{Spatial Autocorrelation Sensitivity Analysis}

The Moran's I test indicated the presence of a significant positive spatial autocorrelation for the outcome, liver cancer incidence $(I=0.28, p=0.005)$. Comparison of ordinary least squares regression and a spatial lag model found no meaningful effect of spatial autocorrelation on model estimates (Table S1 in Supplementary Material).

\section{Preventive Services}

The number of centers offering preventive services are 80, 89, and 28 , respectively, for hepatitis B testing, treatment, and vaccination; 127 and 128, respectively, for hepatitis C testing and treatment; 23 syringe exchange programs (SEPs), with multiple additional 


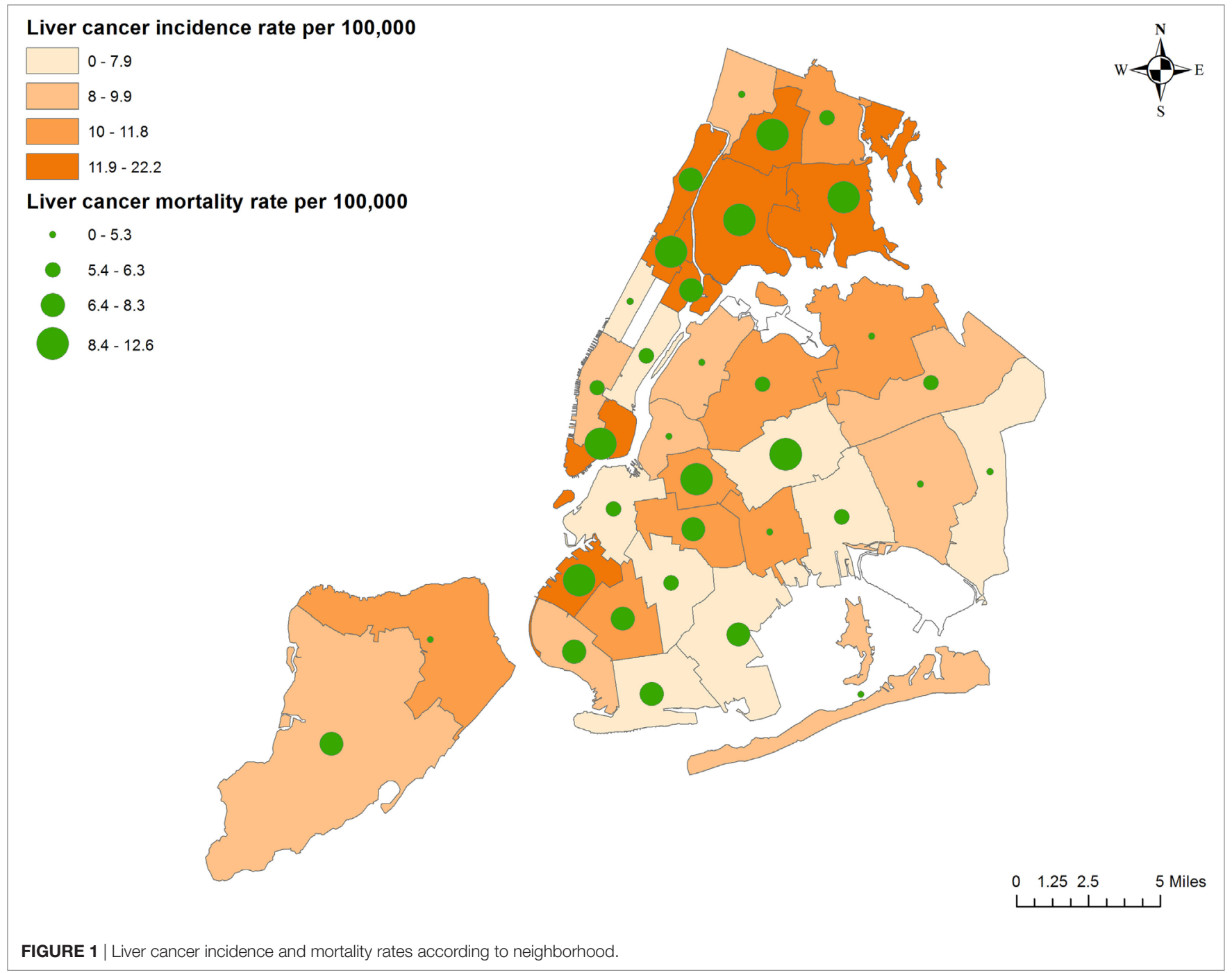

distribution locations, and hundreds of Expanded Syringe Access Program locations throughout NYC. Their availability in relation to hepatitis burden is depicted in Figures 2 and 3.

\section{DISCUSSION}

To the best of our knowledge, this is the first study to describe liver cancer incidence and the distribution of its underlying risk factors at a neighborhood level in NYC. In addition, this is the first study to assess the availability of hepatitis prevention and treatment services in the context of disease burden.

Results indicate that not only does NYC have higher rates of liver cancer incidence and mortality compared with NY State and the rest of the US but also large disparities exist among city neighborhoods, with incidence rates in some neighborhoods as high as those in China and West Africa (7). The most striking finding was the strong relationship between poverty, liver cancer, and its risk factors, even after adjusting for other demographics and risk factor scores. Of the three modifiable risk factor domains, infection was most strongly and consistently associated with liver cancer incidence. Rates of newly reported chronic hepatitis B and C in NYC show a gradual rise since $2013(9,10)$. This could be partially attributed to improved surveillance and test sensitivity and updated US Department of Health guidelines for Hepatitis C testing in "baby boomers" (23). However, recent changes in drug use patterns could explain the rise in both hepatitis B and C rates. The National Institute of Drug Abuse reported that the $\%$ of drug reports identified as heroin, a common injectable drug, increased from $10.4 \%$ in 2012 to $11.6 \%$ in 2013 in NYC, along with a decrease in the average age at admission to substance abuse treatment (24).

Recent immigration patterns may also contribute toward the observed increase in hepatitis B. Between 2000 and 2011, NYC has seen a $4 \%$ increase in foreign-born residents (25). Neighborhoods with the highest gains ( $\geq 5,000$ people) include East and Central Harlem, Lower Manhattan, parts of the South Bronx, Sunset Park, etc. (25). The same neighborhoods have seen high immigration from three countries with high prevalence of hepatitis B (China: 5.49\%, Dominican Republic: 4.09\%, and 
TABLE 2 | Distribution of behavioral liver cancer risk factors according to neighborhood.

\begin{tabular}{|c|c|c|c|c|c|c|c|c|c|c|}
\hline \multirow[t]{2}{*}{ Neighborhood } & \multicolumn{9}{|c|}{ Prevalence of risk factor ( $\%$ of population) ${ }^{h}$} & \multirow{2}{*}{$\begin{array}{l}\text { In the highest } \\
\text { quartile }^{g}\end{array}$} \\
\hline & $\begin{array}{l}\text { Current } \\
\text { smoking }^{\text {a }}\end{array}$ & IDU & Exercise $^{a}$ & $\begin{array}{l}\text { Walked/ } \\
\text { biked }^{\mathrm{a}}\end{array}$ & Obesity $^{a}$ & Diabetes $^{a}$ & $A 1 C \geq 9 \%$ c,d & $\begin{array}{c}\geq 1 \mathrm{HBV} \\
\text { vaccine } \\
\text { dose }^{\mathrm{e}}\end{array}$ & $\begin{array}{l}\text { Ever HCV } \\
\text { tested }^{f}\end{array}$ & \\
\hline Kingsbridge/Riverdale & 8.3 & 0 & 83.4 & 73.9 & 33.3 & 8.7 & 15.1 & 51.7 & 28.3 & \\
\hline The Northeast Bronx & 12.3 & 0 & 72.4 & 78.7 & 28.2 & 10.1 & 17.5 & 58.0 & 54.1 & \\
\hline Fordham/Bronx Park & 10.4 & 1.39 & 71.2 & 80.0 & 28.6 & 18.4 & 20.2 & 47.8 & 46.3 & $L C, M, I, P$ \\
\hline Pelham/Throgs Neck & 16.5 & 0.79 & 73.2 & 76.3 & 29.9 & 11.9 & 19.2 & 51.1 & 42.8 & LC \\
\hline South Bronx $x^{\ominus}$ & 17.0 & 1.76 & 70.4 & 82.1 & 34.4 & 20.2 & 20.7 & 47.1 & 52.4 & LC, M, A, I, P \\
\hline Greenpoint & 20.7 & 0 & 77.9 & 85.1 & 26.2 & 9.4 & 16.2 & 37.8 & 41.0 & \\
\hline $\begin{array}{l}\text { Downtown Brooklyn/Heights/ } \\
\text { Slope }\end{array}$ & 13.5 & 0.64 & 81.2 & 92.0 & 16.1 & 4.6 & 17.1 & 60.7 & 36.1 & \\
\hline $\begin{array}{l}\text { Bedford-Stuyvesant/Crown } \\
\text { Heights }\end{array}$ & 17.9 & 0.23 & 72.8 & 78.1 & 36.3 & 13.6 & 21.1 & 53.7 & 57.2 & $A, P$ \\
\hline East New York/New Lots & 12.3 & 0.19 & 72.3 & 73.5 & 37.1 & 21.7 & 21.5 & 50.1 & 46.5 & $M, A, P$ \\
\hline Sunset Park & 15.3 & 1.51 & 67.1 & 93.1 & 23.6 & 12.1 & 15.6 & 32.9 & 33.4 & $\mathrm{LC}, \mathrm{I}, \mathrm{P}$ \\
\hline Borough Park & 15.1 & 0.48 & 69.8 & 77.8 & 16.3 & 8.8 & 13.4 & 45.3 & 26.7 & 1 \\
\hline Flatbush & 9.4 & 1.03 & 72.9 & 82.0 & 35.6 & 13.7 & 19.6 & 51.1 & 47.3 & \\
\hline Canarsie and Flatlands & 8.0 & 0 & 76.4 & 75.1 & 29.1 & 13.9 & 18.2 & 45.0 & 43.8 & \\
\hline Bay Ridge/Bensonhurst & 15.2 & 0.85 & 73.0 & 83.5 & 21.2 & 10.3 & 12.4 & 33.5 & 30.9 & । \\
\hline Coney Island & 18.4 & 0.93 & 68.4 & 82.6 & 26.8 & 13.7 & 12.5 & 39.9 & 36.2 & I \\
\hline Williamsburg/Bushwick & 18.0 & 1.52 & 69.7 & 79.9 & 25.9 & 15.0 & 21.2 & 42.6 & 46.7 & $M, A, I, P$ \\
\hline Washington Heights/Inwood & 12.0 & 0.44 & 76.8 & 81.2 & 25.8 & 14.1 & 18.6 & 38.0 & 41.9 & LC, M \\
\hline Central Harlem & 12.8 & 0.21 & 74.2 & 81.1 & 31.4 & 13.7 & 19.6 & 40.7 & 45.9 & $L C, I, P$ \\
\hline East Harlem & 16.8 & 4.66 & 65.9 & 83.6 & 27 & 23.1 & 20.7 & 57.3 & 58.3 & $L C, M, A, I, P$ \\
\hline Upper West Side & 13.0 & 2.07 & 91.1 & 90.8 & 7.8 & 6.9 & 14.2 & 63.4 & 37.4 & \\
\hline Upper East Side-Gramercye & 10.2 & 0.4 & 87.6 & 92.1 & 12.5 & 4.1 & 11.3 & 47.6 & 37.4 & \\
\hline Chelsea Village ${ }^{e}$ & 13.2 & 0.6 & 84.2 & 91.3 & 9.3 & 4.8 & 13.1 & 60.6 & 52.1 & 1 \\
\hline Union Sq-Lower Manhattane & 17.0 & 0.19 & 79.8 & 88.4 & 7.9 & 9.3 & 13.3 & 49.9 & 43.9 & LC, A, I \\
\hline Long Island City/Astoria & 19.1 & 0.51 & 80.2 & 85.5 & 25.1 & 9.8 & 15.4 & 42.7 & 29.4 & \\
\hline West Queens & 17.8 & 0.27 & 73.6 & 89.4 & 21.1 & 9.6 & 16.0 & 35.5 & 30.3 & 1 \\
\hline Flushing/Clearview & 11.5 & 0.25 & 67.7 & 79.2 & 17.2 & 10.7 & 11.3 & 45.1 & 43.3 & \\
\hline Bayside-Fresh Meadows ${ }^{e}$ & 9.1 & 0.17 & 75.1 & 78.4 & 19.1 & 14.2 & 10.4 & 53.3 & 30.9 & \\
\hline Ridgewood/Forest Hills & 18.8 & 0 & 70.5 & 83.8 & 17.5 & 5.1 & 13.0 & 40.9 & 36.7 & \\
\hline Southwest Queens & 12.8 & 1.38 & 67.3 & 72.6 & 28.6 & 20.4 & 17.6 & 42.8 & 37.6 & \\
\hline Jamaica & 6.7 & 0.05 & 67.1 & 79.8 & 30.8 & 13.6 & 17.3 & 40.2 & 44.5 & \\
\hline Southeast Queens & 11.0 & 0 & 74.2 & 69.1 & 26.0 & 12.2 & 16.7 & 54.0 & 45.5 & \\
\hline The Rockaways & 16.0 & 0.85 & 69.5 & 77.3 & 34.1 & 13.9 & 18.4 & 41.0 & 53.4 & $\mathrm{M}, \mathrm{A}$ \\
\hline Northern Staten Islande & 17.5 & 0 & 78.5 & 74.2 & 23.7 & 9.0 & 17.9 & 45.2 & 41.1 & \\
\hline Southern Staten Island ${ }^{e}$ & 17.5 & 0.37 & 79.3 & 65.5 & 24.6 & 6.2 & 12.4 & 47.9 & 33.1 & \\
\hline NYC & 14.3 & 0.66 & 74.5 & 81.6 & 24.1 & 11.6 & 17.0 & 46.6 & 41.4 & \\
\hline
\end{tabular}

$L C$, liver and bile duct cancer incidence rate; $M$, metabolic risk factor score; $A$, alcohol risk factor score; I, infection risk factor score; $P$, poverty.

aNew York City (NYC) Department of Health and Mental Hygiene. Epiquery: NYC Interactive Health Data System-[Community Health Survey (CHS) 2015] [08/29/2017].

http://nyc.gov/health/epiquery.

'Injecting drug use: NYC Department of Health and Mental Hygiene. CHS [2012]; public use dataset accessed on 09/08/2017.

"NYC A1C Registry, 2012; rates based on registrants reported with likely diabetes (based on a history of $\geq 2$ A1C test values $\geq 6.5 \%$ ).

${ }^{\prime} N Y C$ residents ages $\geq 18$ years; rates are per 100,000 adults and are age-adjusted to 2000 Census (July 2013 NYSDH population estimates).

${ }^{\circledR} \mathrm{A} 1 \mathrm{C}$ registry data for combined neighborhoods are averages of constituent UHF 42 neighborhoods.

${ }^{f}$ NYC Department of Health and Mental Hygiene. Epiquery: NYC Interactive Health Data System - [CHS 2012] [09/08/2017]. http://nyc.gov/health/epiquery.

${ }_{9}$ NYC Department of Health and Mental Hygiene. Epiquery: NYC Interactive Health Data System - [CHS 2013] [09/08/2017]. http://nyc.gov/health/epiquery.

${ }^{h}$ All percentages are age-adjusted using the 2010 US Census standard population.

Jamaica: 3.76\%) (26), mirroring their own high hepatitis B rates. Chinese-born immigrants in NYC were found to have high seroprevalence of hepatitis B and increased risk for liver cancer (27). Another study found hepatitis B prevalence of 9.6\% among a sample of African-born participants residing mostly in Central Harlem and the South Bronx (28). Immigrant health is an important public health issue in a diverse city like NYC. Most liver cancer risk factors are preventable, but due to poverty or other issues, health policies may not have the desired effect. Ongoing surveillance for hepatitis and effective and timely culturally and linguistically competent prevention and treatment may be the key to preventing progression to liver cirrhosis and liver cancer in NYC residents. The population of certain areas in Harlem and Bronx is ideal for exploring preventive public health strategies, and implementing surveillance programs.

Preventive and treatment services for hepatitis are available throughout the city, but not all neighborhoods with high hepatitis rates have a proportionate number of required services. We observed that high hepatitis B rates were correlated with lower vaccine coverage and lower proportion of free vaccination 
TABLE 3 | Association between liver cancer incidence and risk factor scores.

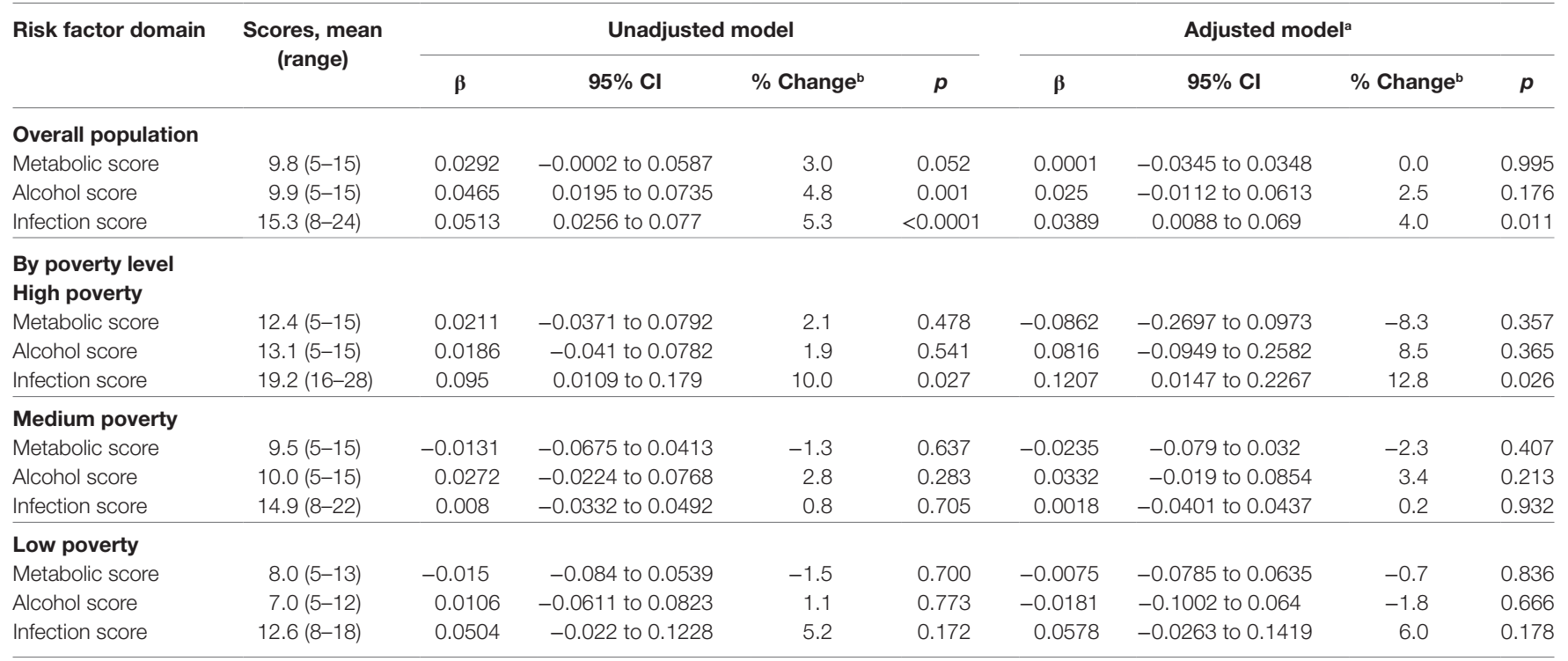

${ }^{a}$ All adjusted models include the following variables: metabolic score, alcohol score, and infection score.

${ }^{b}$ Percentage change in incidence of liver cancer per unit increase in risk score: calculated as $\left(e^{\beta}-1\right) \times 100$.

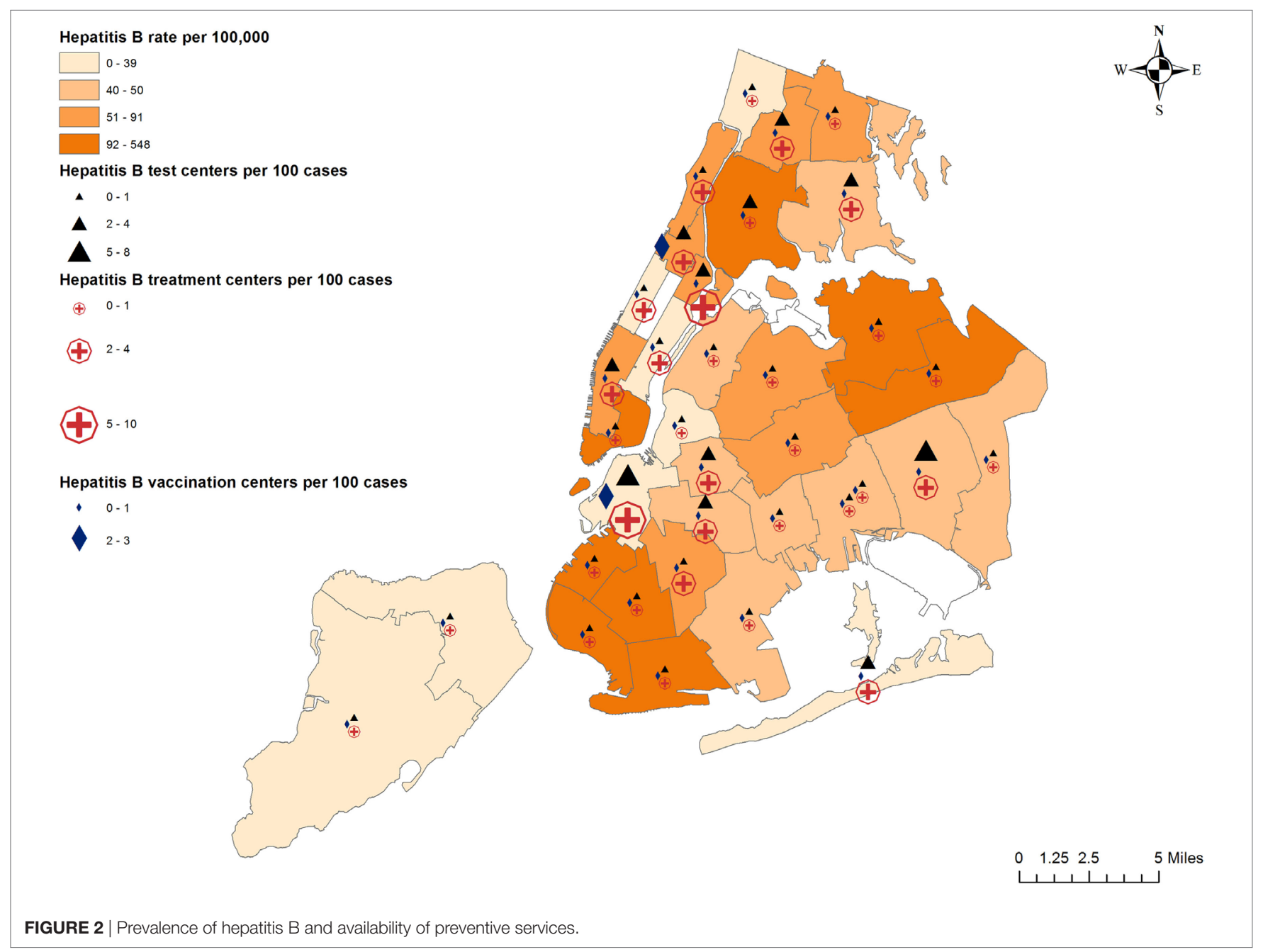




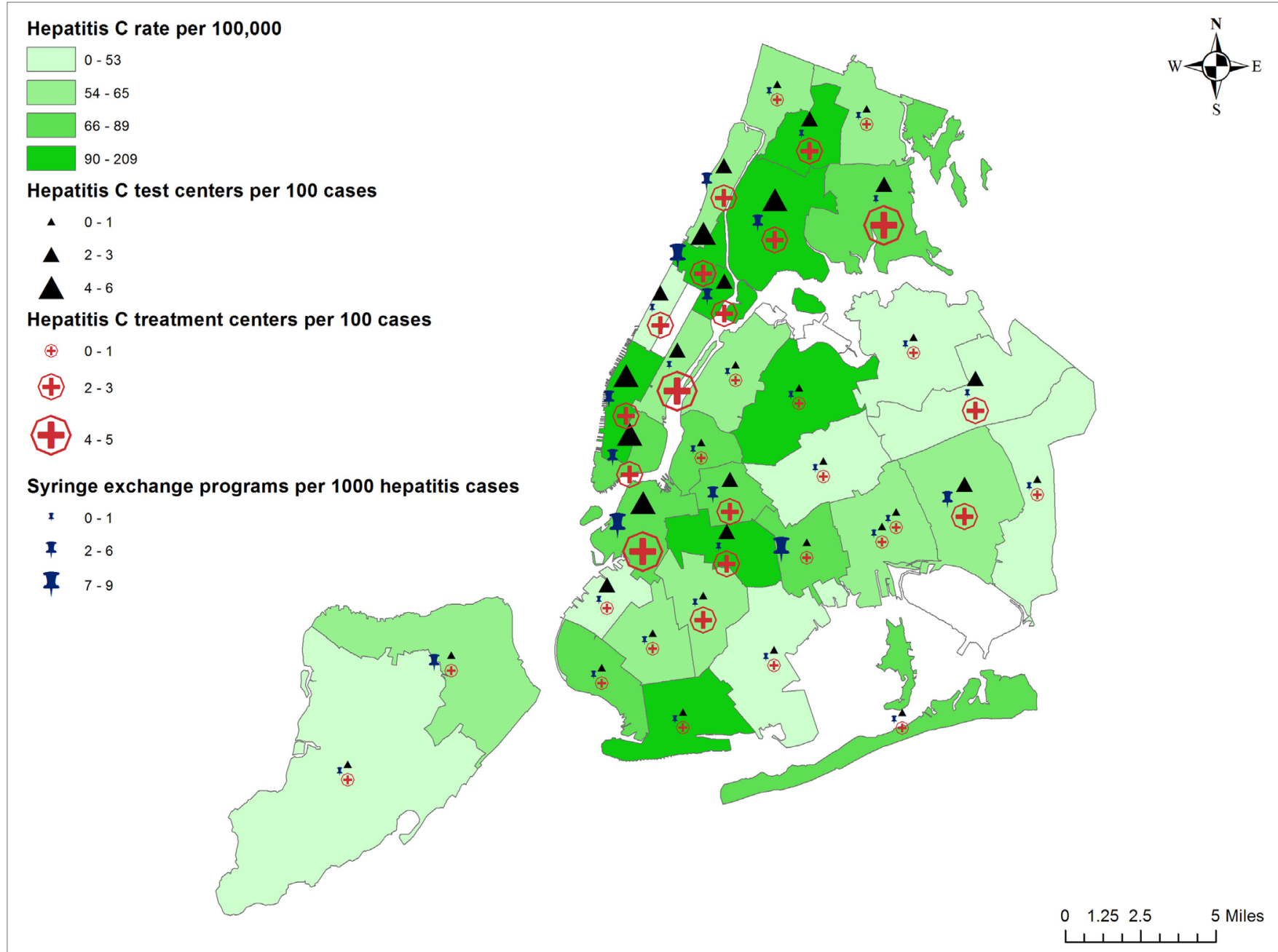

FIGURE 3 | Prevalence of hepatitis $C$ and availability of preventive services.

centers. Lower insurance coverage was also strongly correlated with lower vaccine coverage. Hepatitis $B$ vaccination can cost \$120-\$370 without insurance, plus consultation/professional administration fees. This is largely unaffordable for less affluent, uninsured people. Non-monetary factors such as having a vaccinated acquaintance, perceived risk of disease, perceived vaccine safety, and provider recommendation may also influence patients' choice to receive the hepatitis B vaccine (29). Therefore, a multi-pronged intervention is required to increase hepatitis B vaccine coverage in NYC, addressing disease-specific knowledge, access, affordability, and psychosocial factors.

While hepatitis C-related services were found to be more numerous, some neighborhoods appear to have fewer than 1 SEP per 1,000 hepatitis cases (Fordham-Bronx Park and BedfordStuyvesant-Crown Heights), while others (Coney Island and West Queens) have fewer than one hepatitis $\mathrm{C}$ testing and treatment centers per 100 hepatitis C cases. Although residents in poorer neighborhoods were more likely to get tested for hepatitis $\mathrm{C}$, there is no information on how many of those who tested positive cleared the virus or received treatment. Without insurance, hepatitis C drugs for a 12-week course can cost between
$\$ 39,600$ and $\$ 94,500(30,31)$. Even with insurance, arranging for prior authorization of hepatitis $C$ treatment is often time consuming and a barrier to patients starting treatment, e.g., most NYS insurance providers require a prescription to be written by or in consultation with a specialist (32). Hepatitis C treatment for those who cannot obtain health insurance is provided by the NYS Hepatitis C Patient Assistance Program HepCAP (33). However, many of them are not eligible for HepCAP, highlighting important gaps in current hepatitis $\mathrm{C}$ management.

This study has some limitations: as an ecological study based on the most recently available data, the neighborhood-level associations may not reflect individual risk of liver cancer; thus the results should be interpreted in a geographical context only. Surveillance data for hepatitis B and C may include people that no longer have active infection, and therefore these should not be considered incidence or prevalence rates, but simply the number of newly reported cases. Hepatitis may also be underdiagnosed due to the passive nature of surveillance data, since active testing is more costly and resource intense. Study power to detect significant associations could be restricted by small sample size $(n=34)$. Age, sex, and racial/ethnic diversity are other potential 
source of variation; however, due to the non-individual nature of the data and multiple race indicators, it was not possible to adjust for these variables. Alcohol risk scores were moderately correlated with metabolic and infection risk scores; however, results of statistical tolerance tests did not indicate a significant threat of multicollinearity on the model estimates. The NYC Health Map website does not provide data on private medical offices which may provide vaccinations and/or treatment whose information is not publicly available. Finally, we did not have data on homeless or incarcerated populations, who have an even higher risk of hepatitis C (34). However, this is the first study that attempts to quantify the relative role of infection, metabolic factors, and alcohol in HCC risk in a diverse environment such as NYC and highlights current gaps in hepatitis prevention services like syringe exchange and vaccination, that can be addressed by the expansion of existing services. The role of alcohol and metabolic risk factors on liver cancer in NYC warrants further study.

\section{ETHICS STATEMENT}

This study was carried out in accordance with the recommendations of the Program for the Protection of Human Subjects Office (PPHS), Icahn School of Medicine at Mount Sinai. The protocol was approved (under exempt status) by the Institutional Review Board at the Icahn School of Medicine at Mount Sinai. A consent waiver was obtained since the data were de-identified, publicly available data.

\section{REFERENCES}

1. New York State Cancer Registry. Cancer Incidence and Mortality in New York State, 1976-2014. Available from: http://www.health.ny.gov/statistics/cancer/ registry/ (Accessed: October 11, 2017).

2. National Cancer Institute. Surveillance, Epidemiology, and End Results Program (SEER 18 Data). Available from: https://seer.cancer.gov/faststats/ selections.php?\#Output

3. Llovet JM, Zucman-Rossi J, Pikarsky E, Sangro B, Schwartz M, Sherman M, et al. Hepatocellular carcinoma. Nat Rev Dis Primers (2016) 2:16018. doi:10.1038/nrdp.2016.18

4. Flegal KM, Kruszon-Moran D, Carroll MD, Fryar CD, Ogden CL. Trends in obesity among adults in the United States, 2005 to 2014. JAMA (2016) 315:2284-91. doi:10.1001/jama.2016.6458

5. Heimbach JK, Kulik LM, Finn R, Sirlin CB, Abecassis M, Roberts LR, et al. AASLD guidelines for the treatment of hepatocellular carcinoma. Hepatology (2018) 67:358-80. doi:10.1002/hep.29086

6. US Cancer Statistics Working Group. United States Cancer Statistics: 1999-2014Incidence and Mortality Web-Based Report. Atlanta:U.S.Department of Health and Human Services, Centers for Disease Control and Prevention and National Cancer Institute (2017). Available from: https://nccd.cdc.gov/ uscs/ (Accessed: October 11, 2017).

7. Ferlay J, Soerjomataram I, Dikshit R, Eser S, Mathers C, Rebelo M, et al. Cancer incidence and mortality worldwide: sources, methods and major patterns in GLOBOCAN 2012. Int J Cancer (2015) 136:E359-86. doi:10.1002/ijc.29210

8. Islami F, Miller KD, Siegel RL, Fedewa SA, Ward EM, Jemal A. Disparities in liver cancer occurrence in the United States by race/ethnicity and state. CA Cancer J Clin (2017) 67:273-89. doi:10.3322/caac.21402

9. Hashim D, Farhat Z, Wallenstein S, Manczuk M, Holcombe RF, Thorpe L, et al. Standardized cancer incidence disparities in Upper Manhattan New York City neighborhoods: the role of race/ethnicity, socioeconomic status, and known risk factors. Eur J Cancer Prev (2016) 25:349-56. doi:10.1097/ CEJ.0000000000000180

\section{AUTHOR CONTRIBUTIONS}

GK, ET, and NB had full access to all the data in the study and take responsibility for the integrity of the data and the accuracy of the data analysis. Study concept and design; study supervision: ET and NB. Acquisition, analysis, or interpretation of data: GK, NE, and SE. Drafting of the manuscript: GK, ET, NB, PP, JW, and MS. Critical revision of the manuscript for important intellectual content: GK, ET, NB, NE, PP, JW, MS, JL, and SE. Statistical analysis: GK.

\section{ACKNOWLEDGMENTS}

We thank the New York City Department of Health and Mental Hygiene and the New York State Department of Health for their data.

\section{FUNDING}

This work was supported by a grant from the National Cancer Institute at the National Institutes of Health [P30 CA196521].

\section{SUPPLEMENTARY MATERIAL}

The Supplementary Material for this article can be found online at https://www.frontiersin.org/articles/10.3389/fonc.2018.00220/ full\#supplementary-material.

10. New York City Department of Health and Mental Hygiene. United Hospital Fund (UHF 34) Neighborhood Index. Available from: https://a816-healthpsi. nyc.gov/epiquery/CHS/uhf-zip-information.pdf (Accessed: October 11, 2017).

11. New York City Department of Health and Mental Hygiene. EpiQuery: NYC Interactive Health Data. Available from: https:/a816-healthpsi.nyc.gov/ epiquery/ (Accessed: October 11, 2017).

12. United States Census Bureau. American Community Survey (ACS). Available from: https://www.census.gov/programs-surveys/acs/ (Accessed: October 11, 2017).

13. New York City Department of Health and Mental Hygiene. New York City Community Health Survey (CHS). Available from: http://www1.nyc.gov/site/ doh/data/data-sets/community-health-survey.page (Accessed: October 11, 2017).

14. New York City Department of Health and Mental Hygiene. Communicable Disease Surveillance Data (CDSD). Available from: http://wwwl.nyc.gov/site/ doh/data/health-tools/communicable-diseases.page (Accessed: October 11, 2017).

15. New York City Department of Health and Mental Hygiene. Epi Data Tables, No. 36: Diabetes and its Complications. New York, NY: New York City Department of Health and Mental Hygiene (2013).

16. New York State Department of Health. Statewide Planning and Research Cooperative System (SPARCS). Available from: https://www.health.ny.gov/ statistics/sparcs/ (Accessed: October 11, 2017).

17. Chen CM, Yi HY. Trends in Alcohol-Related Morbidity Among Short-Stay Community Hospital Discharges, United States, 1979-2007. Arlington, VA: US Department of Health and Human Services, Public Health Service, National Institutes of Health (2010).

18. Murphy SL. Deaths: Final Data for 1998. National Vital Statistics Reports. (Vol. 48). Hyattsville, MD (2000).

19. New York City Department of Health and Mental Hygiene. NYC Health Map. Available from: https://a816-healthpsi.nyc.gov/NYCHealthMap (Accessed: October 11, 2017). 
20. Moran PA. Notes on continuous stochastic phenomena. Biometrika (1950) 37:17-23. doi: $10.2307 / 2332142$

21. Nikolopoulos G, Bagos P, Lytras T, Bonovas S. An ecological study of the determinants of differences in 2009 pandemic influenza mortality rates between countries in Europe. PLoS One (2011) 6:e19432. doi:10.1371/journal. pone.0019432

22. Berk KN. Tolerance and condition in regression computations. J Am Stat Assoc (1977) 72:863-6. doi:10.1080/01621459.1977.10479972

23. Blumberg EA. MMWR updates on hepatitis B and C. Am J Transplant (2012) 12:3155-6. doi:10.1111/j.1600-6143.2012.04322.x

24. National Institute of Drug Abuse. Epidemiologic trends in drug abuse. Proceedings of the Community Epidemiology Work Group - Highlights and Executive Summary. Rockville, MD (2014).

25. City of New York Department of City Planning. The Newest New Yorkers: Characteristics of the City's Foreign-Born Population. New York, NY: City of New York Department of City Planning (2013).

26. Schweitzer A, Horn J, Mikolajczyk RT, Krause G, Ott JJ. Estimations of worldwide prevalence of chronic hepatitis B virus infection: a systematic review of data published between 1965 and 2013. Lancet (2015) 386:1546-55. doi:10.1016/S0140-6736(15)61412-X

27. PollackHJ, Kwon SC, Wang SH, Wyatt LC, Trinh-Shevrin C, AAHBP Coalition. Chronic hepatitis B and liver cancer risks among Asian immigrants in New York City: results from a large, community-based screening, evaluation, and treatment program. Cancer Epidemiol Biomarkers Prev (2014) 23:2229-39. doi:10.1158/1055-9965.EPI-14-0491

28. Shankar H, Blanas D, Bichoupan K, Ndiaye D, Carmody E, MartelLaferriere V, et al. A novel collaborative community-based hepatitis B screening and linkage to care program for African immigrants. Clin Infect Dis (2016) 62(Suppl 4):S289-97. doi:10.1093/cid/ciw090

29. Samoff E, Dunn A, Vandevanter N, Blank S, Weisfuse IB. Predictors of acceptance of hepatitis B vaccination in an urban sexually transmitted diseases clinic. Sex Transm Dis (2004) 31:415-20. doi:10.1097/01.OLQ.0000130533.53987.78
30. Morris SY. Hepatitis C Treatment Costs: What You Should Know. Healthline (2016). Available from: https://www.healthline.com/health/hepatitis-c-treatment-cost\#2 (Accessed: December 12, 2017).

31. Hepatitis C Support Project: Hcv Advocate. Fair Pricing Coalition Welcomes FDA Approval of AbbVie's New Pangenotypic HCV Combination Mavyret. (2017). Available from: http://hepatitisc.hcvadvocate.org/2017/08/fairpricing-coalition-welcomes-fda-approval-abbvies-new-pangenotypic-hcvcombination-mavyret.html (Accessed: December 12, 2017).

32. Center for Health Law and Policy Innovation: Harvard Law School. Hepatitis C: State of Medicaid Access Report Card. National Viral Hepatitis Roundtable, Harvard Law School (2017).

33. HepCAP. NYS Hep C Patient Assistance Program (NYS DOH). (2015). Available from: https://hepfree.nyc/hep-c-patient-assistance-programhepcap/ (Accessed: December 12, 2017).

34. New York City Department of Health and Mental Hygiene. Hepatitis B and C in New York City 2015 Annual Report. (2016). Available from: https://www1 nyc.gov/assets/doh/downloads/pdf/cd/hepatitis-b-and-c-annual-report2015.pdf (Accessed: December 12, 2017).

Conflict of Interest Statement: JW has received research grant support and served as a consultant for Gilead Sciences, Inc. JL is a consultant for Bayer, BMS, Eisai, Celsion, Eli Lilly and has active funding for research from Bayer, BMS, and Eisai. NB has active funding from Pfizer for research. All the other authors report no conflicts of interest.

Copyright (c) 2018 Kamath, Taioli, Egorova, Llovet, Perumalswami, Weiss, Schwartz, Ewala and Bickell. This is an open-access article distributed under the terms of the Creative Commons Attribution License (CCBY). The use, distribution or reproduction in other forums is permitted, provided the original author(s) and the copyright owner are credited and that the original publication in this journal is cited, in accordance with accepted academic practice. No use, distribution or reproduction is permitted which does not comply with these terms. 